\title{
Microstructure et comportement hydromécanique d'un sol gonflant non saturé
}

\section{O. CUISINIER}

F. MASROURI

Laboratoire Environnement Géomécanique et Ouvrages ENSG-INPL

Rue du DoyenMarcel-Roubault BP 40 54501 Vandouvre-lèsNancy Cedex

Farimah.Masrouri@ensg. inpl-nancy.fr

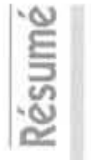

Cet article présente une étude expérimentale du comportement hydromécanique d'un sol gonflant compacté dans une gamme de succion comprise entre 0 et 287,9 MPa. Une première série d'essais a servi à caractériser la variation de différents paramètres hydiromécaniques avec la succion. Elle a permis de montrer que la compressibilité du matériau augmente de manière significative lorsque la succion est réduite de 287,9 à $4 \mathrm{MPa}$, pour se stabiliser en deçà de $4 \mathrm{MPa}$. Ces observations ont été complétées par une étude microscopique de la structure du matériau en fonction de sa succion. La succion de $4 \mathrm{MPa}$ constitue ainsi un seuil en deçà duquel la double structure initiale du matériau étudié est détruite par le gonflement. Ces observations démontrent la forte corrélation qui existe entre le comportement hydromécanique et la structure microscopique d'un matériau gonflant non saturé.

Mots-clés : sol gonflant, sol compacté, succion, paramètres hydromécaniques, compressibilité, microstructure.

This paper presents a study of the hydromechanical behaviour of a compacted swelling soil in the range of suctions comprised between 0 and $287.9 \mathrm{MPa}$. A first test series allowed to characterize the variation of some hydromechanical parameters over the tested suction range: swelling potential, slope of the virgin compression line and preconsolidation pressure. These data were compared with the internal structure of the tested material. This study showed that under $4 \mathrm{MPa}$ the double structure of the soil was destroyed by swelling with the suction decrease. A very good correlation was observed between the mechanical behaviour and the internal structure of the tested material.

Key words: swelling soil, compacted soil, suction, hydromechanical parameters, compressibility, microstructure. 


\section{Introduction}

Le recours à l'utilisation des sols compactés est d'un usage très répandu, en géotechnique ainsi qu'en géotechnique de l'environnement pour l'élaboration de noyau de barrages et pour la réalisation de barrières ouvragées dans les sites de stockage de déchets nucléaires. Dans ces deux derniers cas, les matériaux employés contiennent une part importante de minéraux argileux de la famille des smectites qui présentent la propriété de pouvoir gonfler de manière très importante lors d'une humidification. Après leur mise en place, ces matériaux, initialement non saturés, risquent d'être soumis à des sollicitations hydriques qui peuvent entraîner de fortes modifications de leurs propriétés hydromécaniques associées à des changements importants de leur structure interne. La compréhension des couplages hydromécaniques est un enjeu majeur compte tenu des applications sensibles auxquelles sont destinés ces matériaux.

Pour étudier les phénomènes en jeu, l'utilisation de deux variables indépendantes, définies à partir de la contrainte totale $\sigma$, de la pression de l'air $u_{a^{\prime}}$ et de la pression de l'eau $u$ a été proposée (Coleman, 1962; Matyas et Radakrishna, 1968; Fredlund et Morgenstern, 1977). Il s'agit de la contrainte nette $\sigma^{\prime}=\sigma-u_{n}$ et de la succion $s=u_{-}-u_{u}$

De nombreuses études expérimentales du comportement des sols non saturés ont été réalisées, ce qui a permis de définir un certain nombre de caractéristiques de leur comportement, comme par exemple la diminution de la compressibilité avec l'augmentation de la succion (Alonso et al., 1987 et 1990; Vicol, 1990; Cui, 1993). Cependant, le comportement particulier des sols contenant une quantité importante d'argile gonflante reste assez mal connu en raison, notamment, de difficultés expérimentales. En effet, l'étude des différents paramètres et la caractérisation des couplages hydromécaniques nécessitent la réalisation d'essais permettant d'imposer et de maitriser les sollicitations hydriques, c'est-à-dire les variations de succion. Les techniques expérimentales les plus utilisées pour imposer la succion dans des cedomètres à succion contrôlée, la surpression d'air (Richards, 1935) et la technique osmotique (Kassif \& Ben Shalom, 1971), autorisent uniquement l'imposition de succions comprises entre 0 et quelques MPa. Des évolutions de ces techniques ont été proposées, mais elles ne permettent d'atteindre au maximum que $14 \mathrm{MPa}$ (Villar \& Martin, 1996; Delage et al., 1998). La succion d'un sol gonflant pouvant atteindre plusieurs centaines de $\mathrm{MPa}$, il apparait indispensable d'entreprendre l'étude de ses propriétés hydromécaniques dans le domaine des fortes succions. Actuellement, la seule technique disponible pour atteindre ce domaine est la technique des solutions salines, utilisée pour la première fois dans un cedomètre par Esteban (1990) cité par Villar (1995) et quelques auteurs depuis (Oteo Mazo et al., 1995; Robinet et al., 1997: Al-Mukhtar et al., 1999; Blatz et Graham, 2000; Gasc-Barbier et al., 2000) ce qui a permis d'atteindre une succion de plusieurs centaines de MPa. Afin d'améliorer la connaissance du comportement hydromécanique d'un sol gonflant, le premier but de l'article est de présenter une étude menée sur un tel matériau dans une gamme de succions comprises entre 0 et $287,9 \mathrm{MPa}$. Celle-ci a été réalisée à partir de l'utilisation de plusieurs œedomètres à succion contrô- lée par des solutions salines ou grâce à la méthode osmotique.

Les matériaux gonflants compactés ont une organisation interne particulière, qualifiée de double structure par Gens et Alonso (1992). En effet, les particules argileuses s'agglomerent avec les autres constituants du sol pour former des agrégats. Il y a donc dans ces sols des macropores situés entre les agrégats et des micropores au sein des agrégats. Cette structure est extrêmement dépendante de l'état hydrique du matériau, et évolue au cours du processus d'humidification (Cui et al., 2002). Or, il n'existe qu'un nombre restreint de publications s'intéressant à l'influence de la structure d'un matériau compacté sur son comportement mécanique (Delage \& Lefebvre, 1984; Qi et al., 1996; Guillot et al., 2001). Dans ce contexte, le troisième objectif de cet article est d'introduire une étude de la microstructure d'un matériau gonflant en fonction de son état hydrique, et de corréler ces données à son comportement hydromécanique.

\section{2}

\section{Techniques expérimentales utilisées}

Deux types d'œedomètre à succion contrôlée ont été mis en place pour cette étude.

\section{1}

\section{Cidomètre à succion imposée par la méthode osmotique}

Le principe de la méthode est de mettre en contact une éprouvette et une solution de macromolécules, et d'insérer entre les deux une membrane semi-perméable. Celle-ci empêche le passage des macromolécules de la solution vers l'éprouvette mais elle permet le passage de l'eau. Les échanges d'eau, et donc la succion, sont contrôlés par le phénomène d'osmose: plus la concentration en macromolécules est grande, plus la succion est forte, La macromolécule utilisée classiquement est le polyéthylèneglycol (PEG). De plus amples détails sur cette technique sont disponibles dans. Williams et Shaykewich (1969), Cui (1993) et Cuisinier (2002). Delage et al. (1998) et Cuisinier \& Masrouri (2001) ont démontré que cette méthode pouvait être utilisée dans la gamme des succions comprises entre 0 et $8,5 \mathrm{MPa}$.

L'cedomètre osmotique utilisé au cours de cette étude est présenté sur la figure 1. Avec plusieurs paliers de chargement, la durée moyenne d'un essai dans un œdomètre osmotique est comprise entre 2 et 3 mois.

\section{2}

\section{๔domètre à succion imposée par solutions salines saturées}

Le principe de ce type d'œdomètre (Fig. 2) est d'enfermer un système équivalent à une cellule œdométrique classique dans une enceinte dont l'humidité relative est maintenue constante grâce à une solution saline saturée. L'humidité relative $\mathrm{Hr}$ est reliée à la succion s, par la loi de Kelvin:

$$
s=\gamma_{w} \frac{\mathrm{RT}}{\mathrm{Mg}} \ln \left(\frac{\mathrm{p}}{\mathrm{p}_{0}}\right)
$$




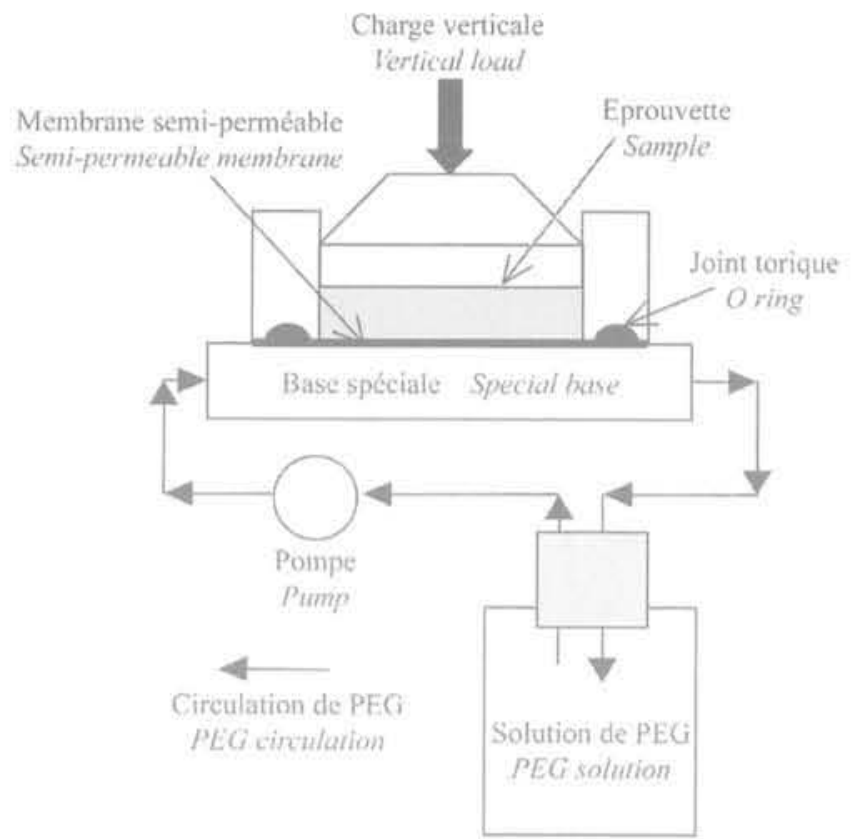

FG. 1. Eedomètre à succion contrôlée par la méthode osmotique.

Suction controlled oedometer using the osmotic technique.

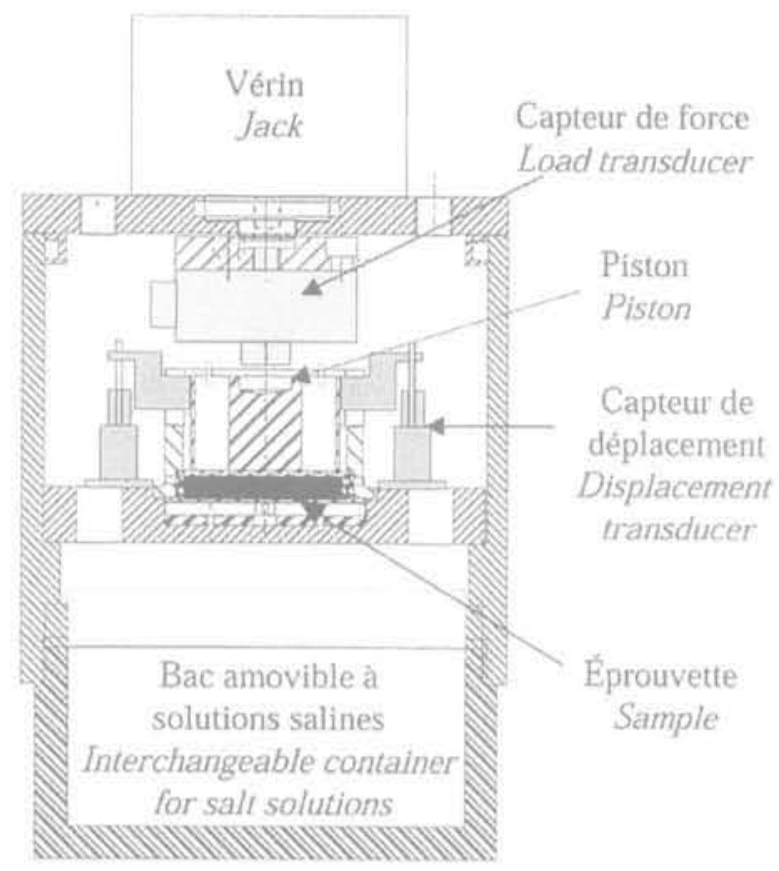

FIG. 2 Edomètre à succion contrôlée par solutions salines.

Suction controlled oedometer using salt solutions.

avec $\gamma_{\text {u }}$ le poids volumique de l'eau $\left(9,81 \mathrm{kN} \cdot \mathrm{m}^{-3}\right), \mathrm{g}$ Taccélération de la pesanteur, $\mathrm{M}$ la masse molaire de l'eau $\left(18.10^{-3} \mathrm{~kg} \cdot \mathrm{mol}^{-1}\right)$ et $\mathrm{R}$ la constante des gaz parfaits $\left(8,31 \mathrm{~J} \cdot \mathrm{mol}^{-1} \cdot \mathrm{K}^{-1}\right), \mathrm{p}_{0}$ la pression de vapeur saturante à la température absolue $\mathrm{T}(\mathrm{kPa})$ et $\mathrm{p}$ la pression partielle de vapeur ( $\mathrm{kPa}$ ). L'utilisation de plusieurs solutions salines saturées permet d'imposer différentes succions, la valeur de l'humidité relative imposée par un sel donné ayant été obtenue dans Lide (2002) et AFNOR (1999). Cette méthode peut être utilisée dans la gamme des succions supérieures à 8,5 MPa (Delage et al., 1998; Cuisinier \& Masrouri, 2001).

Deux nouveaux appareils (Fig. 2), avec deux capacités d'application de pression verticale maximale différentes (1 200 ou $20000 \mathrm{kPa}$ ), ont été développés (Cuisinier \& Masrouri, 2003). Avec plusieurs paliers de chargement, un seul essai peut durer plusieurs mois afin d'atteindre les équilibres hydrique et mécanique.

\section{3}

\section{Matériau étudié et préparation des éprouvettes}

Le matériau étudié est un mélange de $40 \%$ de limon de Xeuilley et de $60 \%$ de bentonite calcique. Les principales caractéristiques de ces matériaux sont indiquées dans le tableau 1 .

Le limon et la bentonite, initialement sous forme de poudre tamisée à $400 \mu \mathrm{m}$, sont mélangés, puis humidifiés à une teneur en eau de $15 \%$. Cette valeur, proche de la limite de retrait, a été sélectionnée de manière à limiter la rétraction de l'éprouvette lors d'une dessiccation. Les éprouvettes sont préparées par compactage statique sous une pression de $1 \mathrm{MPa}$. Leur hauteur est de $1 \mathrm{~cm}$ et leur diamètre vaut 7,4 ou $5 \mathrm{~cm}$ dans les œdomètres à solutions salines et $7 \mathrm{~cm}$ dans les œedo-

TABLEAUI Caractéristiques des matériaux étudiés. Caracteristics of the studied materials.

\begin{tabular}{lccc}
\multicolumn{1}{c}{\begin{tabular}{c} 
Matériau \\
\multicolumn{1}{c}{ étudié }
\end{tabular}} & $w_{6}$ & Ip & $\gamma$ \\
Limon & $(\%)$ & $(\%)$ & $\left(\mathrm{kN}^{-3} \mathrm{~m}^{-3}\right)$ \\
Bentonite & 42 & 5 & 27 \\
Mélange & 117 & 31 & 26 \\
& 87 & 21 & 26
\end{tabular}

mètres osmotiques. Le poids volumique sec initial est d'environ $12,7 \mathrm{kN} \cdot \mathrm{m}^{-3}$. La succion totale des éprouvettes a été mesurée par la technique du papier filtre (ASTM, 1995b). Elle est comprise entre 20 et $25 \mathrm{MPa}$.

Le potentiel de gonflement, qui correspond au rapport entre la variation de hauteur de l'éprouvette provoquée par une humidification et sa hauteur initiale, ainsi que la pression de gonflement, qui est la pression nécessaire pour éliminer la variation de hauteur produite par l'humidification, ont été déterminés par fa méthode du gonflement libre (ASTM, 1995a), Ces grandeurs sont de $17 \%$ et de $250 \mathrm{kPa}$ respectivement.

\section{4}

\section{Comportement hydromécanique entre 0 et $287,9 \mathrm{MPa}$}

La figure 3 et les tableaux II et III permettent de visualiser précisément tous les chemins de contrainte dans le plan $\left(\sigma^{\circ}-\mathrm{s}\right)$. L'état initial est repéré par le point «A $)$, La référence de chaque essai indique le type d'appareil utilisé ( $\mathrm{S}$ solutions salines et $\mathrm{O}$ osmotique), puis le type de chemin suivi ( $\mathrm{W}$ correspond à une phase d'humidification, $\mathrm{D}$ à une phase de dessiccation et $\mathrm{L}$ à une phase de chargement). L'essai OWL6, réalisé sous une succion nulle, n'a pas pu être représenté sur la figure 3. 
TABLEAU II Description des chemins suivis (méthode des solutions salines) [ $\mathrm{S}$ : méthode des solutions salines: W: humidification; D : dessiccation; L : chargement/déchargement],

Stress paths description (salt solutions technique) [S: salt solutions technique; W: wetting: D: drying;

L: loading/unloading].

$\begin{array}{cccccc}\text { SWL1 } & \text { SDL1 } & \text { SDL2 } & \text { SDL3 } & \text { SDL4 } \\ \text { A-K-L-K } & \text { A-B-A } & \text { A-C-D-C } & \text { A-C-E-F-E } & \text { A-C-G-H-G }\end{array}$

TABLEAU III Description des chemins suivis (méthode osmotique) [S: méthode des solutions salines :

W: humidification; D : dessiccation ; L : chargement/déchargement].

Stress paths description (osmotic method) iS : salt solutions technique; W: wetting: D: drying:

L. loading/unloading].

$\begin{array}{ccccc}\text { OWL1 } & \text { OWL2 } & \text { OWL3 } & \text { OWL4 } & \text { OWL5 } \\ \text { A-K-L-K } & \text { A-K-M-N-M } & \text { A-K-M-O-P-O } & \text { A-K-M-Q-R-Q } & \text { A-K-O-S-T-S }\end{array}$

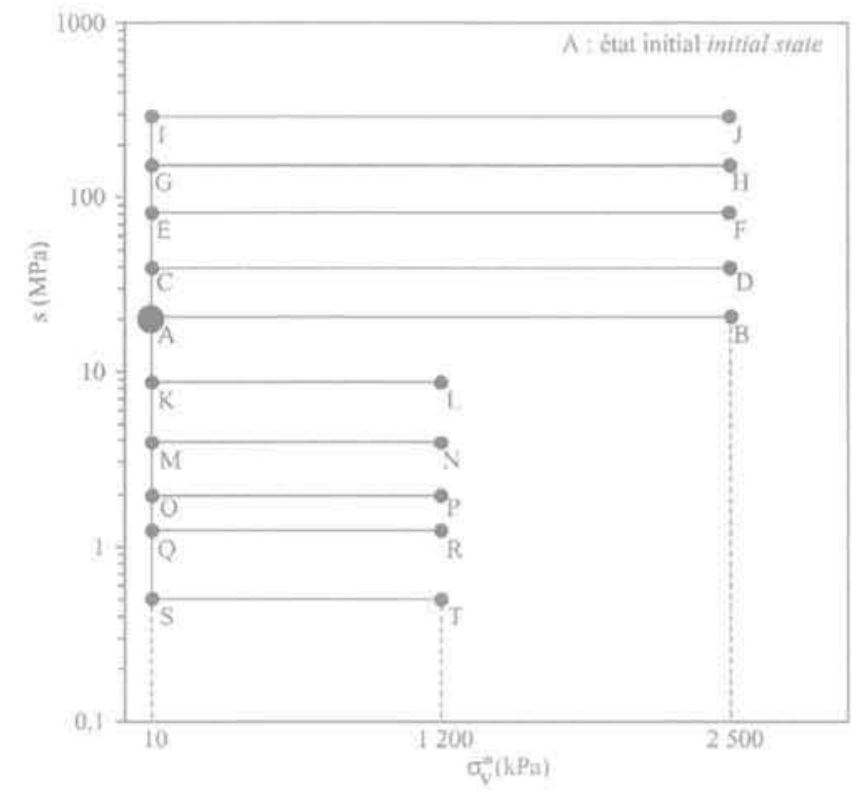

FG. 3 Chemins de contrainte suivis. Stress paths followed.

A partir de chaque courbe expérimentale obtenue, différents paramètres ont été déterminés. Il s'agit du gonflement lors de la phase d'application de la succion sous faible charge verticale $\Delta \mathrm{H} / \mathrm{H}$, de la pente de compression plastique $\lambda(\mathrm{s})$, de la pente de rebond élastique $\kappa$ et de la contrainte de préconsolidation $\mathrm{p}_{0}(\mathrm{~s})$. Dans le cas des sols non saturés, la contrainte de préconsolidation dépend de la plus grande pression connue par le sol ainsi que de la valeur de sa succion. Pour cette raison, le terme de contrainte de préconsolidation apparente sera conservé dans la suite de cet article.

\section{1}

\section{Validation des dispositifs expérimentaux}

Les résultats des essais ayant servi à la validation des dispositifs expérimentaux sont reportés sur la figure 4 et dans le tableau IV.

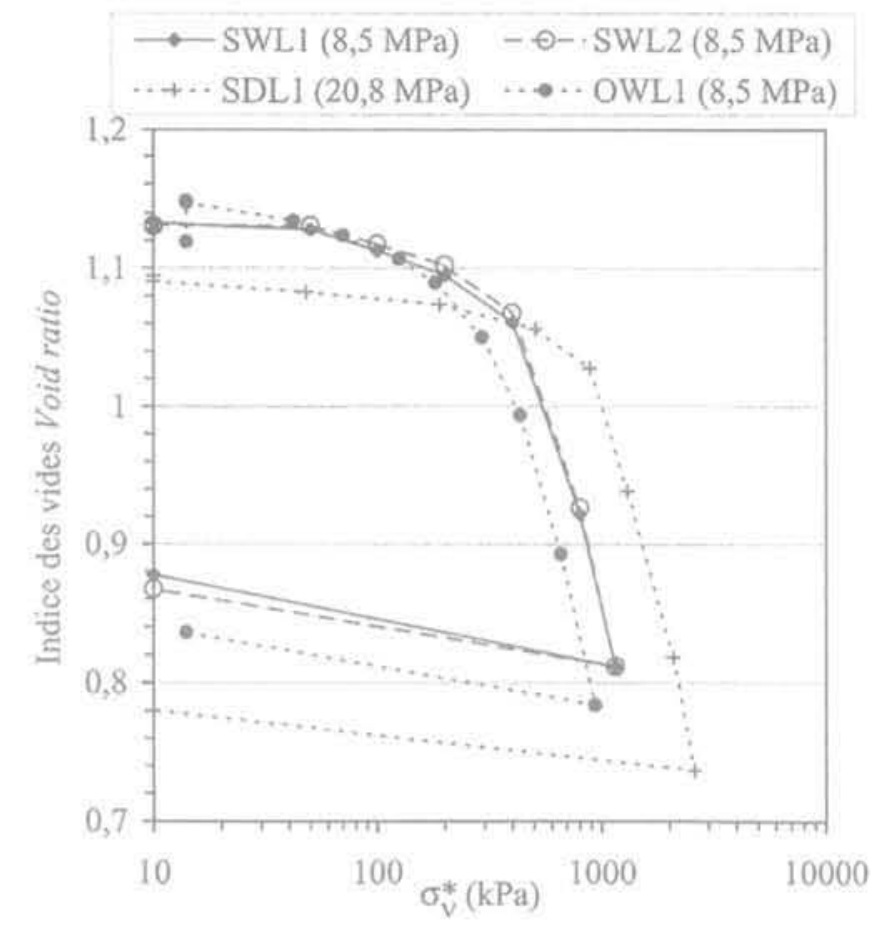

FIG. 4 Résultats des essais utilisés pour valider les dispositifs expérimentaux.

Results of the tests conducted to validate the experimental devices.

L'essai SDL1, oủ la succion imposée est voisine de la succion initiale, permet de retrouver la contrainte de préconsolidation imposée lors de la préparation des éprouvettes par compactage statique, soit $1000 \mathrm{kPa}$. Cet essai valide donc le principe d'utilisation des solutions salines saturées pour imposer la succion dans un œdomètre. 
Deux essais, SWL1 et SWL2, ont été réalisés en respectant le même chemin de contrainte de manière à contrôler la répétabilité des essais. Les deux courbes œdométriques obtenues sont quasiment identiques et de plus, les paramètres hydromécaniques calculés (tableau IV) sont similaires dans les deux cas. La répétabilité dans les appareils à solutions salines est donc satisfaisante.

La succion totale d'un sol est la somme de plusieurs composantes, les principales étant la composante matricielle et d'adsorption et la composante osmotique (Fredlund et Rahardjo, 1993). Or, la technique des solutions salines permet d'imposer la succion totale alors que la méthode osmotique n'autorise que la maittrise de la succion matricielle. La question se pose donc de savoir si, pour la succion limitant le domaine d'utilisation des deux méthodes, la continuité entre les deux techniques est acceptable. Pour répondre à cette question, deux essais ont été réalisés sous une succion de $8,5 \mathrm{MPa}$, un dans chaque type d'œdomètre (SWL1 et OWL1) (Fig. 4). Les résultats obtenus montrent que la pression $\mathrm{p}_{\mathrm{f}}(\mathrm{s})$ et le gonflement sont légèrement affectés par la technique de contrôle de succion tandis que les pentes $\lambda(s)$ et $\kappa$ ne sont pas significativement influencées. L'utilisation complémentaire de ces deux techniques est donc sujette à quelques limitations, la technique osmotique et la méthode des solutions salines ne permettant pas d'imposer les mêmes composantes de la succion. Il est vraisemblable que si la continuité avait été testée sous une succion appliquée plus faible, la composante osmotique d'un sol augmentant avec le degré de saturation, les différences de comportement observées auraient été amplifiées. Dans le cas du matériau testé, la succion de $8,5 \mathrm{MPa}$ semble adaptée pour que les effets de la composante osmotique de la succion sur le comportement mécanique ne soient pas trop importants.

\section{2}

\section{Propriétés hydromécaniques}

Cette étude a été réalisée à partir de douze essatis hydromécaniques qui débutent tous par une phase de modification de la succion sous contrainte verticale faible constante $(10 \mathrm{kPa})$. Ensuite, un chargement/ déchargement œedométrique à succion constante est réalisé. Il s'agit des essais dont les résultats sont reportés sur les figures 5 et 6 ainsi que dans le tableau IV. Ces figures montrent que le comportement du maté- riau étudié varie fortement sur l'ensemble de la gamme de succion. Les courbes de compressibilité peuvent être organisées selon deux groupes distincts. Lorsque la succion appliquée est inférieure ou égale à $2 \mathrm{MPa}$, les courbes de compressibilité correspondantes tendent vers la courbe de compressibilité obtenue sous une succion nulle (essai OWL6). La pression pour laquelle elles rejoignent la courbe de l'essai OWL6 est d'autant plus forte que la succion appliquée est élevée. Le deuxième groupe de courbes correspond aux essais où la succion imposée est supérieure à $2 \mathrm{MPa}$, ces courbes de compressibilité traversant la courbe de l'essai OWL6 sans la rejoindre. La courbe de compressibilité obtenue dans le cas de l'essai OWL2 ( $\mathrm{s}=4 \mathrm{MPa}$ ) est particulière dans le sens où elle traverse la courbe de l'essai OWL6 mais les deux se rejoignent à partir d'une pression verticale appliquée de $550 \mathrm{kPa}$ environ.

Lors de l'humidification, deux phases de gonflement peuvent ètre identifiées (Fig. 7). Dans le cadre des essais présentés ici, la limite entre cés deux phases peut être fixée à une succion comprise entre 4 et $2 \mathrm{MPa}$ environ. En effet, lorsque la succion est réduite de 4 à $2 \mathrm{MPa}$ le gonflement augmente de 4 à $5 \%$ alors que lorsque la succion est diminuée de $2 \mathrm{MPa}$ jusqu'à $1,2 \mathrm{MPa}$, la valeur du gonflement passe de 5 à $10 \%$ environ. La phase de faible gonflement pourrait s'expliquer par un comblement progressif des vides de l'éprouvette, le gonflement macroscopique devenant très important lorsqu'il ne reste plus de vides disponibles à l'intérieur de l'éprouvette.

Ces données peuvent être comparées à la variation de la pression $\mathrm{p}_{0}(\mathrm{~s})$ avec la succion afin d'évaluer l'influence du gonflement sur ce paramètre (Fig. 7). On constate que dans la phase de faible gonflement que la pression $\mathrm{p}_{0}(\mathrm{~s})$ diminue de 1000 à $200 \mathrm{kPa}$ alors que le gonflement est limité. Ce point montre l'importance des phénomènes microscopiques sur le comportement mécanique d'un matériau gonflant. On assiste à une stabilisation de la pression $\mathrm{p}_{0}(\mathrm{~s})$ au-dessus d'une succion de $38,9 \mathrm{MPa}$ (Tableau IV).

La pente $\kappa$ diminue lorsque la succion augmente (Fig. 8). La pente $\lambda(\mathrm{s})$ varie de façon non monotone avec ta succion. En effet, entre une succion de $4 \mathrm{MPa}$ et $287.9 \mathrm{MPa}$ la pente $\lambda(\mathrm{s})$ diminue lorsque la succion augmente. Entre 0 et $4 \mathrm{MPa}$, la pente $\lambda(\mathrm{s})$ est quasi constante et égale à la pente $\lambda(\mathrm{s})$ lorsque la succion est nulle (essai OWL6). Or, dans la plupart des données existantes dans la bibliographie, la pente $\lambda(\mathrm{s})$ est supposée diminuer avec l'augmentation de succion puis atteindre dans le domaine des très fortes succions une valeur constante (Alonso et al., 1999; Gens et Alonso, 1992).

TABLEAU IV Paramètres hydromécaniques des éprouvettes utilisées.

Compressibility parameters of the tested samples.

\begin{tabular}{|c|c|c|c|c|c|}
\hline Essai & $\begin{array}{c}\text { Succion appliquée } \\
\text { (MPa) }\end{array}$ & $\Delta H / H(\%)$ & $\kappa$ & $\lambda(s)$ & $\begin{array}{l}\mathrm{P}_{0}(\mathrm{~s}) \\
(\mathrm{KPa})\end{array}$ \\
\hline SDL5 & 287,9 & $-3,1$ & 0,005 & 0,09 & 1200 \\
\hline SDL4 & 150,6 & -2.1 & 0,01 & 0,14 & 1200 \\
\hline SDL3 & 83,6 & $-1,7$ & 0,03 & 0,24 & 1200 \\
\hline SDL2 & 38,9 & -0.4 & 0,03 & 0,26 & 1150 \\
\hline SDL1 & 20,5 & 0 & 0,03 & 0,27 & 1000 \\
\hline SWL1 & 8.5 & 0,5 & 0,03 & 0,31 & 450 \\
\hline OWL1 & 8,5 & 1,2 & 0,03 & 0,31 & 370 \\
\hline OWL2 & 4 & 3,9 & 0,03 & 0,30 & 200 \\
\hline OWL3 & 2 & 4,5 & 0,03 & 0,25 & 110 \\
\hline OWL4 & 1.2 & 9.5 & 0,03 & 0,23 & 65 \\
\hline OWL5 & 0,5 & 10,4 & 0,06 & 0,23 & 65 \\
\hline OWL6 & 0 & 17,2 & 0,07 & 0,23 & 55 \\
\hline
\end{tabular}




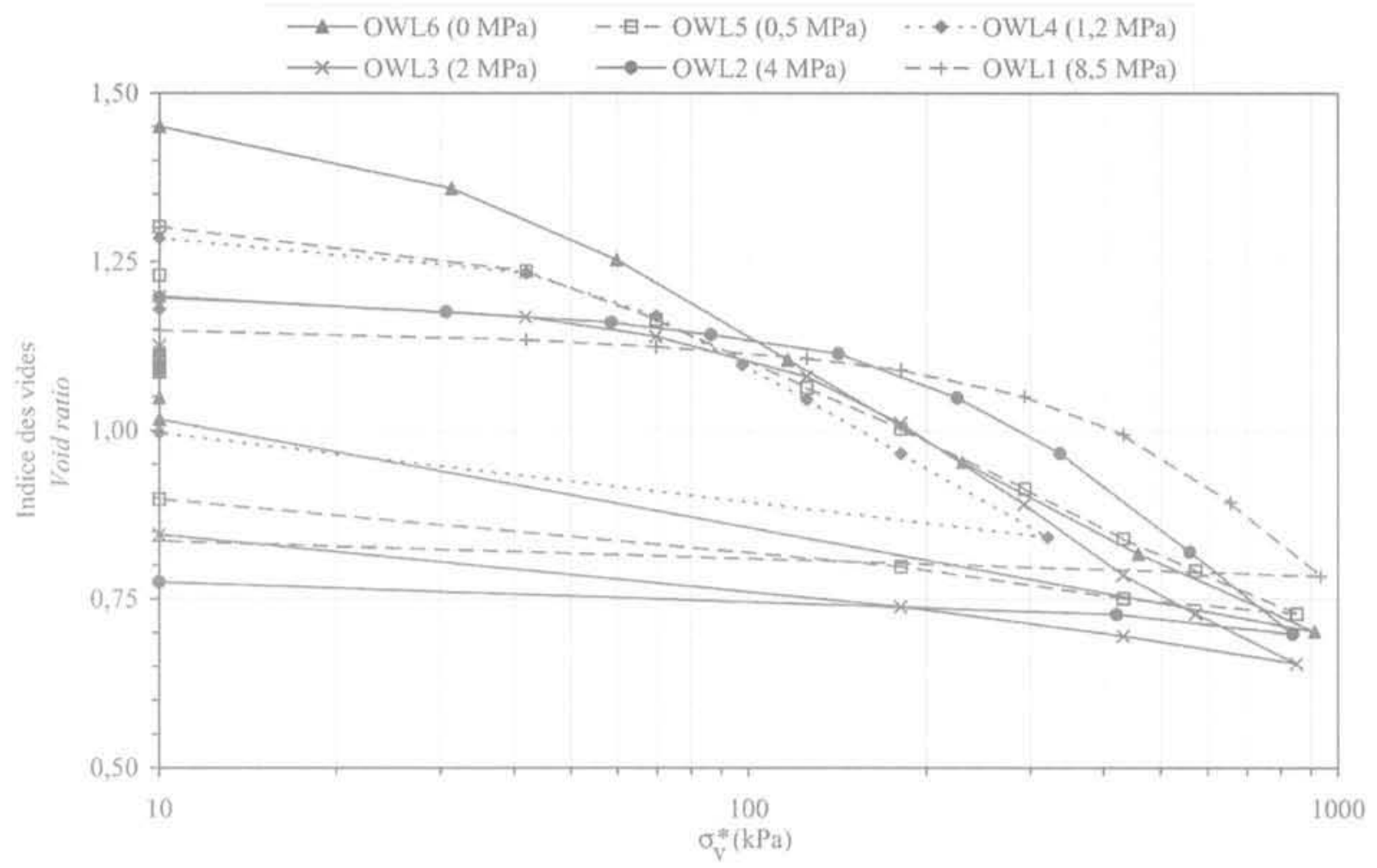

FG. 5 Compressibilité en fonction de la succion appliquée (œedomètre osmotique). Compressibility as a function of the applied suction (osmotic oedometer).

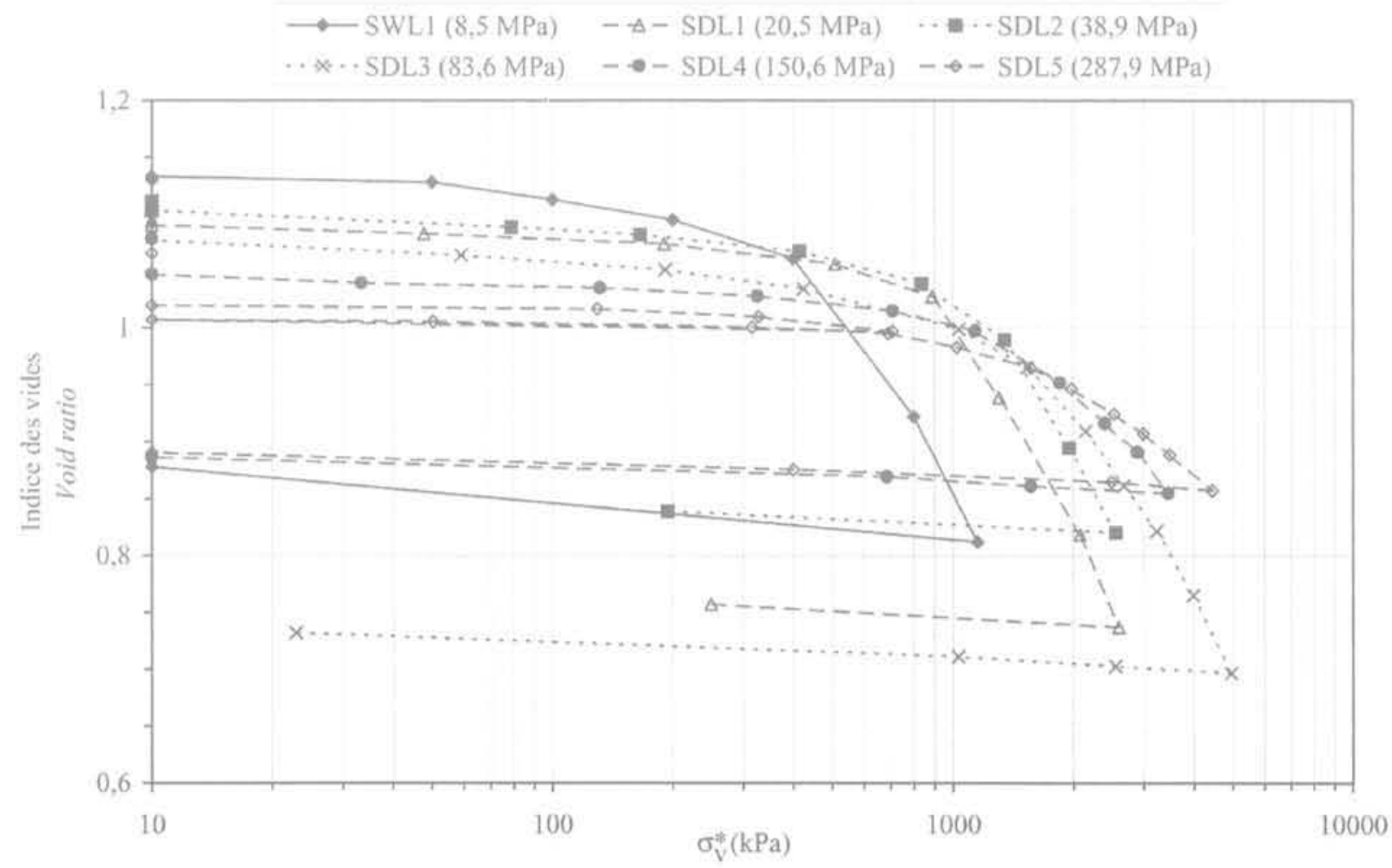

FG. 6 Compressibilité en fonction de la succion appliquée (œedomètre à solutions salines). Compressibility as a function of the applied suction (salt solutions oedometer). 


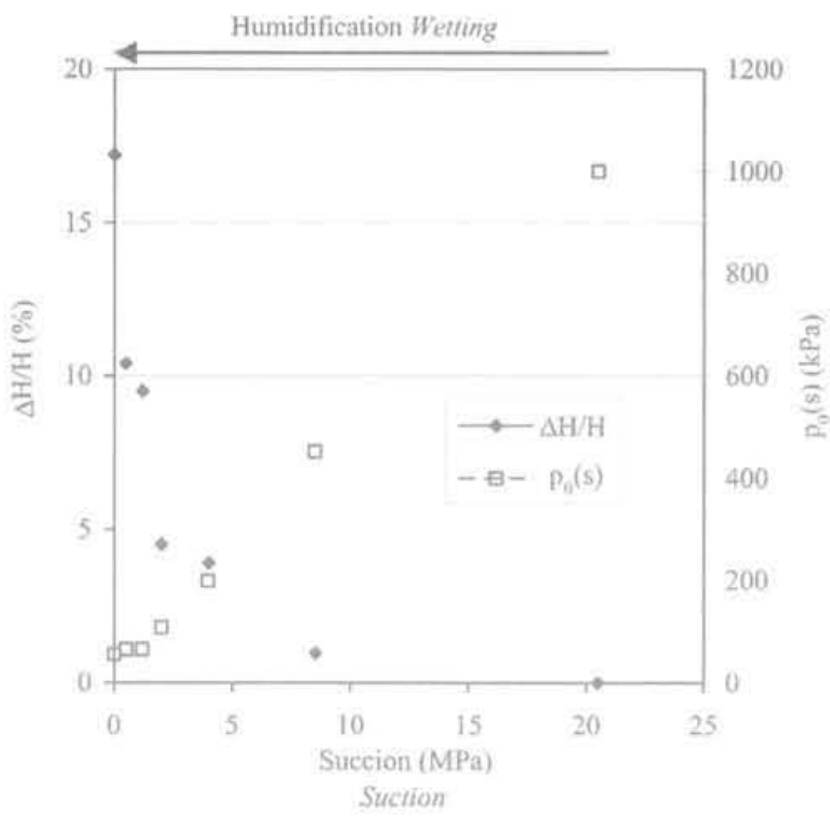

Fis.7 Gonflement et pression de préconsolidation apparente lors de l'humidification. Swelling and apparent preconsolidation pressure during wetting.

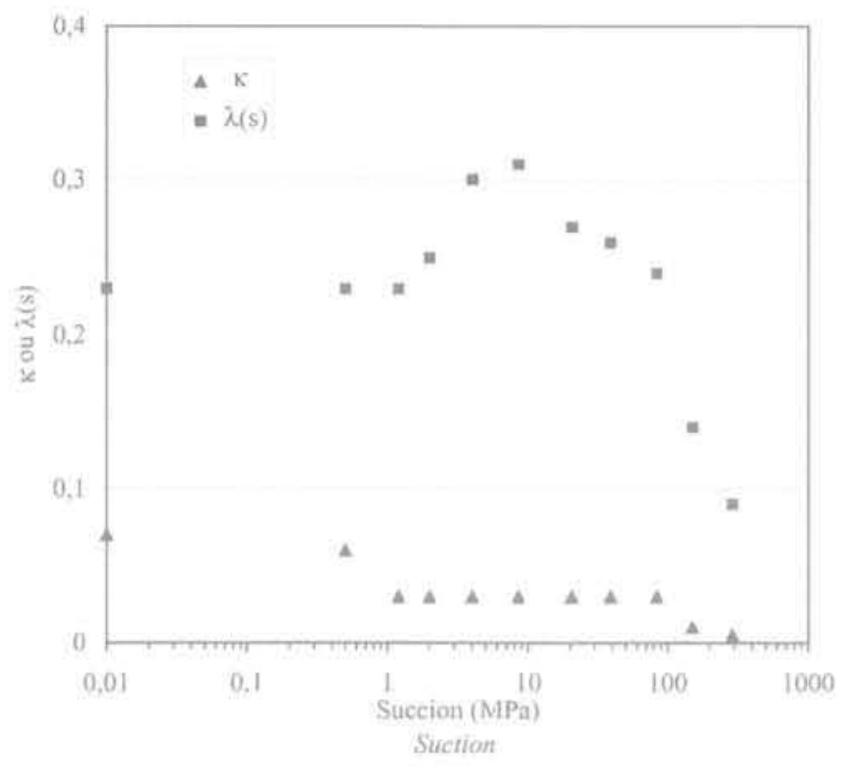

FG. 8 Pentes de compression élastique et plastique.

Elastic and plastic compression slopes.

Un comportement similaire à celui mis en évidence sur le mélange a été observé notamment par Wheelèr \& Sivakumar (1995) qui travaillaient sur un kaolin et Geiser (1999) qui utilisait un limon, c'est-à-dire deux matériaux non gonflants. A partir du même kaolin, Sivakumar et Wheeler (2000) et Wheeler et Sivakumar (2000) ont montré que ce comportement particulier disparaissait lorsque l'énergie de compactage était augmentée, l'évolution de la pente $\lambda(\mathrm{s})$ avec la succion étant alors identique aux données classiques existantes. Les auteurs supposent que le paramètre $\lambda(\mathrm{s})$ dépend de la structure initiale interne des éprouvettes testées qui est fonction de la pression et de la teneur en eau ayant servi pour le compactage des éprouvettes. Geiser (1999) remarque que le pic observé sur la courbe $\lambda($ s) en fonction de la succion correspond au point d'entrée d'air du matériau.

Ces résultats montrent que le comportement du matériau étudié change de manière significative de part et d'autre d'une succion de $4 \mathrm{MPa}$. Dans ce contexte, afin d'évaluer la corrélation entre comportement mécanique et structure pour le matériau gonflant employé, une étude microscopique de la structure des éprouvettes a été entreprise.

\section{5}

\section{Étude de la structure interne des éprouvettes}

Dans cette partie, les résultats de l'étude de la structure interne des éprouvettes en fonction de leur état hydrique sont présentés. Ces observations sont ensuite comparées au comportement mécanique mis en évidence précédemment.

\section{1}

\section{Préparation des lames minces}

La technique de préparation retenue consiste à faire pénétrer, en plusieurs étapes, à l'intérieur de fragments d'éprouvette une résine époxy. L'infiltration de cette résine se déroule selon les étapes suivantes:

- prélèvement de fragments d'éprouvette qui sont ensuite disposés dans de la gélose liquéfiée par un chauffage préalable (température $<50^{\circ} \mathrm{C}$ ). Cette gélose se solidifie en refroidissant, ce qui permet d'éviter les variations de volume du fragment lors des phases de préparation ultérieures et les modifications de structure;

- introduction du fragment dans une solution d'acétone diluée avec de l'eau, l'acétone ayant tendance à pénétrer dans le sol maintenu par la gélose. Cette étape est répétée plusieurs fois, la concentration en acétone étant augmentée progressivement jusqu'à obtenir une solution d'acétone pure:

- introduction des fragments dans une solution d'acétone et de résine époxy (faible concentration de résine). Suivant le même principe que précédemment, la concentration en résine est augmentée progressivement jusqu'à $100 \%$;

- polymérisation de la résine époxy par chauffage et préparation des lames minces.

Cette résine, une fois polymérisée, permet de "fixer» la structure interne des éprouvettes en durcissant. Cette technique autorise la réalisation de lames minces de notre matériau suffisamment fines pour être exploitables en microscopie optique (Foster et De, 1971). Avec cette méthode on peut considérer que la structure interne de l'éprouvette n'est pas perturbée de manière significative lors de la préparation (Kim et al., 1995; Elsass et al., 1998).

La structure interne des éprouvettes a été étudiée sous trois succions différentes: $0 \mathrm{MPa}, 4 \mathrm{MPa}$ et la succion initiale (20 MPa). Trois éprouvettes ont été préparées à une teneur en eau initiale voisine de $15 \%$ dont deux directement dans les œdométres osmotiques. Ces deux dernières éprouvettes ont ensuite été humidifiées 
en plusieurs paliers, chacune jusqu'à une succion différente $(0$ et $4 \mathrm{MPa}$ ). La valeur de $4 \mathrm{MPa}$ a été choisie car elle est proche de la valeur du point d'entrée d'air estimé et elle correspond à la succion à partir de laquelle la réduction de succion provoque un gonflement significatif. Durant l'humidification, le gonflement était libre dans la direction verticale. Un fragment a été prélevé sur chacune des trois éprouvettes, puis préparé pour l'observation microscopique.

\section{2}

\section{Observations microscopiques}

Les résultats obtenus sont donnés sur les fiqures 9 , 10 et 11. L'état initial (Fig. 9), avec une succion comprise entre 20 et $25 \mathrm{MPa}$, est caractérisé par l'existence de macropores, qui individualisent des agrégats associant minéraux argileux et autres constituants du sol. Ceci correspond typiquement à une double structure. L'éprouvette préparée à une succion de $4 \mathrm{MPa}$ montre une structure interne relativement similaire à ce qui est. observé à l'état initial avec cependant une diminution importante de la taille des macropores ainsi que de leur nombre. L'éprouvette préparée à une succion de $0 \mathrm{MPa}$ possède en revanche une structure totalement différente, la totalité des macropores ayant disparu l'éprouvette est composée d'une matrice argileuse homogène dans laquelle il est difficile d'identifier des pores.

On observe donc un comblement progressif des macropores au cours de l'humidification. Cette étude permet de confirmer que la phase de faible gonflement correspond bien au comblement des macropores par les particules argileuses en cours d'expansion, phénomène aussi observé par Cui et al. (2002) sur une argile gonflante mais dans des essais réalisés à volume constant. On peut supposer que tant que le sol se situe dans cette phase de faible gonflement, le matériau a une double structure qui doit donc disparaitre lorsque le gonflement macroscopique devient significatif, c'està-dire entre 4 et $2 \mathrm{MPa}$. Au-dessous de $2 \mathrm{MPa}$, la structure interne de l'éprouvette doit être assez proche de ce qui est observé à $0 \mathrm{MPa}$ (Fig. 11).

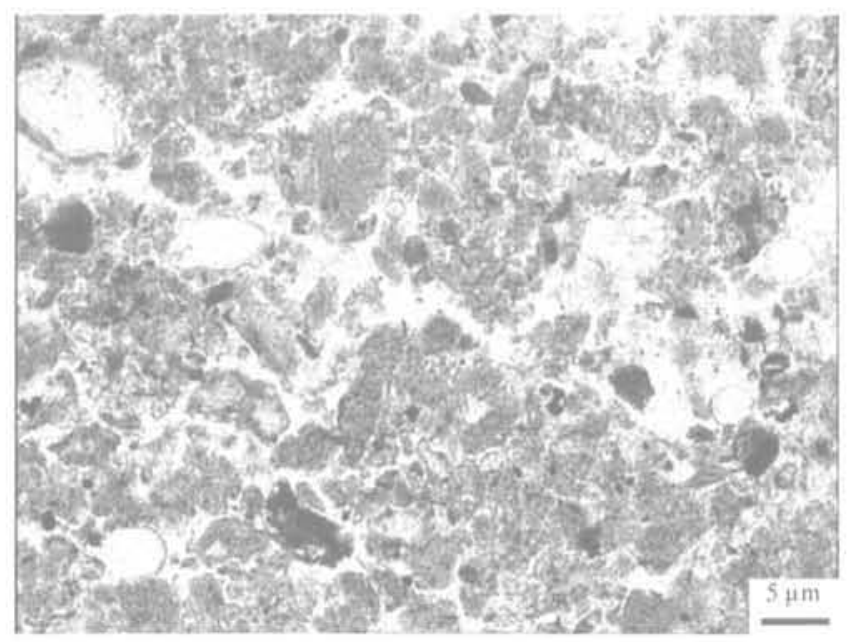

FIG.9 Structure interne d'une éprouvette après compactage $(s=20 / 25 \mathrm{MPa})$ sur lame mince (microscope optique).

Internal structure of a sample thin section after compaction ( $\mathrm{s}=20 / 25 \mathrm{MPa}$ ) (light microscope).

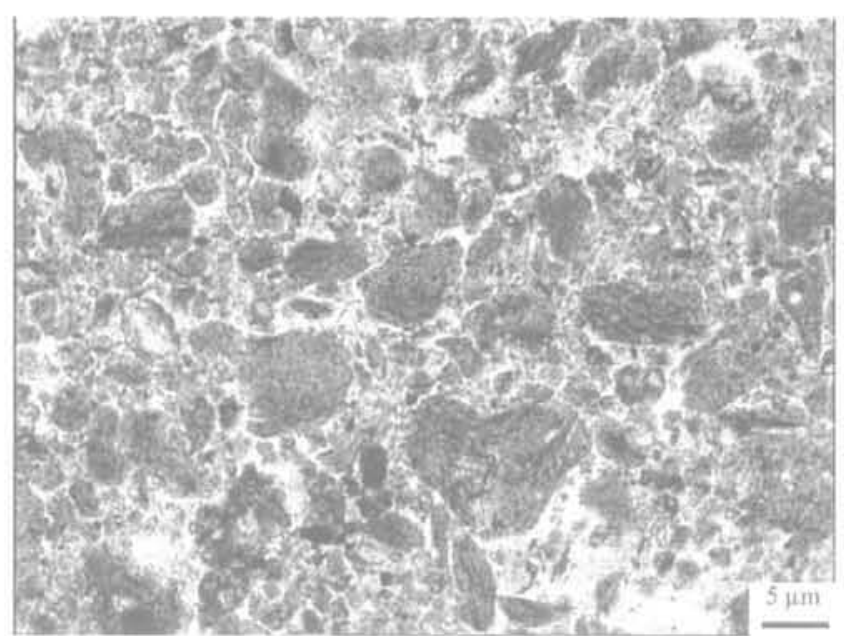

FIG.10 Structure interne d'une éprouvette sous une succion de $4 \mathrm{MPa}$ sur lame mince (microscope optique).

Internal structure of a sample thin section under a suction of $4 \mathrm{MPa}$ (light microscope).

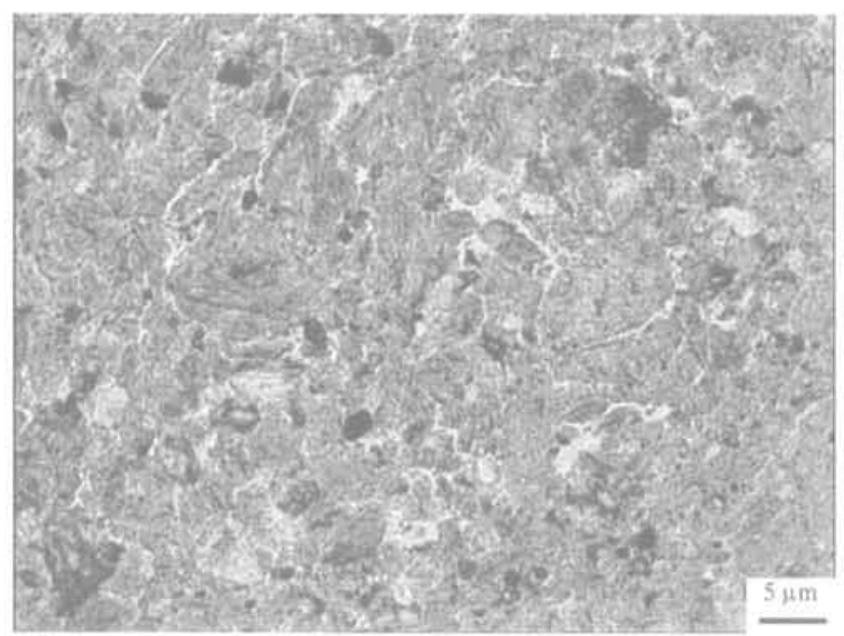

FIG.11 Structure interne d'une éprouvette sous une succion de $0 \mathrm{MPa}$ sur lame mince (microscope optique).

Internal structure of a sample thin section under a suction of $0 \mathrm{MPa}$ (light microscope).

\section{Discussion}

Cette étude de la structure interne des éprouvettes en fonction de leur état hydrique permet de constater que les deux domaines de comportement mécanique mis en évidence sont chacun associés à une structure particulière du matériau. A partir de cette observation. il est possible d'avancer quelques hypothèses sur la relation entre comportement mécanique et structure interne. L'augmentation de la pente $\lambda(\mathrm{s})$ qui traduit une augmentation de la compressibilité lorsque la succion est diminuée de 287,9 à $4 \mathrm{MPa}$ serait le résultat de l'augmentation de la compressibilité des agrégats qui s'humidifient et gonflent. Ce gonflement des agrégats entraine une modification des contacts entre chacun d'eux, ce qui est à l'origine de la réduction de la pression $\mathrm{p}_{0}(\mathrm{~s})$ dans cette gamme de succion. Dans le domaine des succions comprises entre 4 et $0 \mathrm{MPa}$, il est 
possible que le comportement hydromécanique du mélange soit gouverné par les interactions entre les particules argileuses. Cette hypothèse semble confirmée par les résultats de Guiras-Skandaji (1996) qui a travaillé sur la bentonite pure, les éprouvettes étant préparées suivant la mème procédure que celle utilisée ici. La valeur de la pente $\lambda(\mathrm{s})$ sous une succion nulle pour la bentonite pure déterminée par cet auteur est en effet identique à la valeur de $\lambda$ (s) du mélange pour une succion nulle (essai OWL6).

Ces résultats permettent de montrer que le comportement mécanique de ce matériau est fortement corrélé à sa structure interne, qui est donc un élément essentiel pour la compréhension du comportement hydromécanique des sols gonflants compactés.

7

\section{Conclusion}

Cette étude a tout d'abord permis la validation de deux œedomètres à succion contrôlée utilisant la technique des solutions salines saturées et la méthode osmotique. Les essais conduits ont permis de montrer l'importance de la technique d'imposition de la succion sur le comportement mécanique du matériau testé lorsque la succion imposée au cours du chargement mécanique est de 8,5 MPa. L'utilisation combinée des deux techniques semble sujette à quelques précautions, elle pourrait être entreprise lorsque la composante osmotique du matériau est suffisamment limitée.
Ensuite, une étude du comportement hydromécanique d'un matériau gonflant compacté a été réalisée dans le domaine des succions entre 0 et $287,9 \mathrm{MPa}$. Elle a permis de montrer que les paramètres hydromécaniques d'un sol gonflant variaient de manière non monotone sur l'ensemble de la gamme de succion. L'étude présentée a permis de montrer que la structure interne du matériau change de manière importante avec sa succion, ces changements étant fortement corrélés au comportement hydromécanique macroscopique du matériau, tant au niveau de ses propriétés mécaniques ( $\left.\lambda(s), p_{0}(s)\right)$ que de ses caractéristiques hydriques (courbe de rétention).

L'ensemble de cette étude démontre l'extrême sensibilité des propriétés hydromécaniques des sols gonflants à toute variation de succion. La mise en cuvre de ces matériaux gonflants étant envisagée dans des contextes d'utilisation très sensibles, il semble important de poursuivre les investigations entreprises. Cellesci devront porter sur une meilleure définition des couplages hydromécaniques à partir de la réalisation d'essais comportant plusieurs cycles hydriques d'amplitudes variables, sous différentes contraintes verticales ainsi que des états initiaux des éprouvettes variables.

\section{REMERCIEMENTS}

Les auteurs tiennent à remercier pour les expériences d'observation microscopique Monsieur Bartoli. Directeur de Recherche au CNRS, et Madame Villemin, Ingénieur de Recherche au CNRS, appartenant tous deux au Laboratoire Sol et Environnement, UMR 1120. ENSAIA-INPL, Nancy, France.

\section{Bibliographie}

AFNOR NF X 15-119 - Mesure de l'humidité de l'air. 1999, 22 p.

Al-Mukhtar M., Qi Y., Alcover J.F., Bergaya F. - "Oedometric and water retention behavior of highly compacted unsaturated smectites $n$. Revue canadienne de géotechnique, vol. 36, 1999, p. 675 . 684

Alonso E.E., Gens A., Hight D.W. - «Spe cial problem soils n. General report. Proc. of the 9th European Conf. on Soil Mechanics and Foundation Engineering, 1987, p. 5.1-5.60.

Alonso E.E., Gens A. Josa A. - "A constítutive model for partially saturated soils w. Géotechnique, ${ }^{\circ} 40,1990$, p. 405 430.

Alonso E.E., Vaunat J., Gens A. - « Model. ling the mechanical behaviour of expansive clays n. Engineering Geology, vol. 54. 1999, p. 173-183.

ASTM D 4546-90. - "One-dimensional swell or settlement potential of cohesive soils in. Vol. 4.08, 1995a, p. 693-699.

ASTM D 5298-94. - Measurement of soil potential (suction) using filter paper ". Vol. 4.09, 1995b, p. 154-159.

Blatz J., Graham, J. - $\alpha$ A system for controlled suction in triaxial tests 1). Géotechnique, vol. 50, 2000, p. 465-478.

Coleman J.D. - "Stress-strain relations for partiy saturated soils », Gẻotechnique, vol. 12,1962 , p. 348-350.
Cui Y.J. - Etude du comportement d'un limon compacté non saturé et de sa modélisation dans un cadre élastoplastique. Thèse de I'ENPC. 1993. 280 p.

Cui Y.J. Loiseau C. Delage, P. - a Microstructure of a confined swelling soil due to suction controlled hydration n. Proc. 3rdint. Conf. on Unsaturated Soils, Recife, Brésil, vol. 2, 2002, p. 593-598.

Cuisinier 0. - Comportement hvdromécanique des sols gonflant compactés. Thèse de l'INPL., 2002, 200 p

Cuisinier O.. Masrouri F. - " Stucty of the hydromechanical behaviour of a swelling soil from low to very high suctions 3. 6th Int. Workshop on Key Issues in Waste Isolation Research. Paris, 2001. p. 61-70.

Cuisinier O., Masrouri F. - «Comportement hydromécanique d'un sol gonflant sous très fortes succions in. Comptes rendus Mécanique, vol.331, 2003, p. 203-210.

Delage P., Howat M. D., CuI Y.J. - "The relationship between suction and swel. ling properties in a heavily compacted unsaturated clay $¥$. Engineering Geology, vol. 50, 1998, p. 31-48

Delage P. Lefebvre G. - " Study of the structure of a sensitive Champlain clay and of its evolution during consolidation $x$. Revue canadienne de géotechnique, vol. 21, 1984, p. 21-35.

Elsass F., Beaumont A., Pernes M., Jaunet
A.M., Tessier D. - " Changes in layer organization of $\mathrm{Na}$ - and $\mathrm{Ca}$-exchanged smectites during solvent exchanges for embedment resin 1 . The Canadian Mineralogist. vol. 36, 1998, p. 1475-1483.

Foster R.H., De P.K. - w Optical and electron microscopic investigation of shear induced structures in lightly consolidated (soft) and heavily consolidated (hard) kaolinite s. Clays and Clay Minerals, vol. 42, 1971, p. 31-47.

Fredlund D.G., Morgenstern N.R. "Stress state variables for unsaturated soils n. Journal of Geotechnical Engineering, vol. 103. 1977, p. 447-466.

Fredlund D.G., Rahardjo H. - Soils mechanics for unsaturated soils, John Wiley \& Sons, 1993, 517 p.

Gasc-Barbier M., Cosenza P., Goreychi M.. Chanchole S. Tessier D. - - Conception d'un essai triaxial à succion contrôlée: mesure des déformations x. Comptes rendus de l'Académie des sciences. sciences de la terre et des planètes. vol. 330, 2000, p. 97-103.

Geiser F. - Comportement mécanique d'un limon non saturé. Étude expérimentale et modélisation constitutive. Thèse de I'EPFL, Suisse, 1999, 224 p.

Gens A. Alonso E.E. - "A framework for the behaviour of unsaturated expansive clays ». Revue canadienne de géotechnique, vol. 29, 1992, p. 1013-1032. 
Guillot X., Bergaya F., Al-Mukhtar M. Fleureau J.M. - Influence of stress and suction on volume changes and microtexture of a Ca-smectite 3. Int. Symp. on Suction. Swelling. Permeability and Structure of Clays, 2001, p. 147-152.

Guiras-Skandaii H. - Déformabilité des sols argileux non saturés : étude expérimentale et application à la modélisation. Thèse de liNPL. 1996, 315 p.

Kassif G., Ben Shalom A. - "Experimental relationship between swell pressure and suction s. Géatechnique, vol. 21, 1971. p. $245-255$.

Kim J.W., Peacor D.R. Tessier D., Elsass E. - "A technique for maintaining texture and permanent expansion of smectites interlavers for TEM observations 2. Clays and Clay Minerals, vol. 43, 1995; p. 51 -55.

Lide D. R. (ed). - Handbook of chemistry and physics. CRC press., 2002, $8^{\circ}$ ed.. p. 8.108-8.116 et 15.25-15.26.

Matyas E.L., Radhakrishna H.S. - "Volume change characteristics of partially saturated soils m. Géotechnique, vol. 18, 1968. p. $432-448$.

Qi Y., Al-Mukhtar M., Alcover J.F., Ber- gaya F. - " Coupling analysis of macroscopic and microscopic behaviour in highly consolidated Na-iaponite clays $n$. Applied Clay Science, vol. 11, 19996. p. 185-197.

Oteo Mazo C., Saez Auñon J., Esteban F. u Laboratory tests and equipment with suction control n. Alonso \& Delage (eds), Proc. 1st Int. Conf. on Unsaturated Solis, Paris, vol. 3, 1995, p. 1509-1515.

Richards L.A. - «Capillary conduction of liquids through porous medium s. Physics, vol: 1. 1935, p. 318-333.

Robinet J.C., Pasquiou A., Jullien A., Belanteur N., Plas F. - « Expériences de laboratoire sur le comportement thermo-hydro-mécanique de matériatux argileux remaniès gonflants et non gonflants x. Revue française de géotechnique, $n^{\circ} 81,1997, \mathrm{p} .53-80$.

Sivakumar V., Wheeler S.J, - a Influence of compaction procedure on the mechanical behaviour of an unsaturated compacted clay n. Part 1: « Wetting and isotropic compression... Geotechnique, vol. 50. 2000, p. 359-368.

Vicol T. -Comportement hydiraulique et mécanique dun sol fin non saturé. Appli- cation à la modélisation. Thèse de I'ENPC, 1990, 257 p.

Villar M.V. - a First results of suction controlled oedometer tests in highly expansive montmorillonite w, Proc, 1st Int. Conf. on Unsaturated Soils, Paris. vol. 1. 1995, p. 207-213.

Villar M.V., Martin P.L. - "Suction-control. led oedometer tests in montmorillonite clay: preliminary results n. Engineering Geology of Waste Disposal, vol, 11, 1996. p. $309-312$

Wheeler S.J., Sivakumar V. - "An elastoplastic critical state framework for unsaturated soll x. Géotechnique, vol. 45 , 1995; p. 35-53.

Wheeler S.J.. Sivakumar V. - a Influence of compaction procedure on the mechani. cal behaviour of an unsaturated compacted clay p. Part 2: $\alpha$ Shearing and constitutive modelling 1. Géotechnique. vol. 50,2000 , p. 369-376.

Williams J. Shavkewich C.F. - « An evaluation of polyethylene glycol (PEG) 6000 and PEG 20000 in the osmotic control of soll water matric potential 1 . Canadian Journal of Soil Science, vol. 49, 1969, p. $397-401$ 